\title{
Opioid Use After Hospital Care due to Hip Fracture Among Community-Dwelling Persons With and Without Alzheimer's Disease
}

\author{
Eveliina Lintula ${ }^{1}$ Miia Tiihonen ${ }^{1,2}(1) \cdot$ Heidi Taipale ${ }^{1,2,3,4} \cdot$ Anna-Maija Tolppanen $^{1,2} \cdot$ Antti Tanskanen $^{3,4}$. \\ Jari Tiihonen $^{3,4}$ - Sirpa Hartikainen ${ }^{1,2}$. Aleksi Hamina ${ }^{1,2}$
}

Published online: 27 December 2019

(c) The Author(s) 2019

\begin{abstract}
Background Hip fractures are common among persons with Alzheimer's disease (AD), but problems in pain assessment may lead to insufficient analgesia after hospitalization.

Objective We investigated the prevalence of opioid use in the 6 months after discharge from hospital care due to hip fracture among community-dwellers with and without AD.

Setting and method The Medication use and Alzheimer's disease (MEDALZ) cohort was used for this study, consisting of all community-dwelling persons newly diagnosed with AD during 2005-2011 in Finland and their comparison persons without AD matched on age, sex, and region of residence at the time of AD diagnosis. Data were collected from nationwide healthcare registers.

Main outcome measures We investigated opioid use versus non-use in persons with and without AD in the 6 months after discharge from hospital care due to hip fracture.

Results Altogether 2342 persons with AD and 1615 persons without AD, discharged to community settings within $\leq 120$ days after a hip fracture, were included. A higher percentage of persons with AD used opioids compared to those without AD, $39.5 \%$ [95\% confidence interval (CI) 37.5-41.5] versus 31.2\% (95\% CI 28.9-33.5). Persons with AD used more frequently strong opioids during the first 3 months and buprenorphine during the 6-month period, and used weak opioids less frequently after the first month after discharge compared to those without AD.

Conclusions Frequent opioid use after hospital care due to hip fracture may indicate increased attention to pain among persons with AD. Nevertheless, the benefits and harms of opioid use should be evaluated regularly in community-dwelling older persons.
\end{abstract}

\section{Key Points}

Electronic supplementary material The online version of this article (https://doi.org/10.1007/s40266-019-00734-0) contains supplementary material, which is available to authorized users.

Miia Tiihonen

miia.tiihonen@uef.fi

1 School of Pharmacy, University of Eastern Finland, P.O.B 1627, 70211 Kuopio, Finland

2 Kuopio Research Centre of Geriatric Care, University of Eastern Finland, P.O.B 1627, 70211 Kuopio, Finland

3 Department of Clinical Neuroscience, Karolinska Institutet, 17177 Stockholm, Sweden

4 Department of Forensic Psychiatry, Niuvanniemi Hospital, University of Eastern Finland, Niuvankuja 65, 70240 Kuopio, Finland
Persons with Alzheimer's disease used opioids more frequently than persons without Alzheimer's disease after hospital discharge after hip fracture.

Pain and need for opioid use should be evaluated regularly after hospital care.

During opioid use, careful monitoring of the adverse effects and events of opioids is needed. 


\section{Introduction}

Alzheimer's disease (AD) is the most common cognitive disorder and is increasing in prevalence globally as the population ages [1]. One key aspect of health among persons with $\mathrm{AD}$ is falls and subsequent fractures. Persons with $\mathrm{AD}$ are at a high risk of falling and have a two- to threefold increased risk of hip fracture compared to those without $\mathrm{AD}[2,3]$.

Aphasia and decline of cognitive functioning in $\mathrm{AD}$ lead to difficulties in expressing pain verbally, and therefore, the assessment and management of pain may be challenging [4]. In addition, pain can be a contributory cause to neuropsychiatric symptoms, such as aggression and agitation [5], and also for delirium [6]. Previously, suspicions of inadequate pain treatment in persons with $\mathrm{AD}$ and other dementias have been raised, as findings of less frequent analgesic use compare to other older adults have been common [7-10]. However, more recent studies indicate more frequent use of paracetamol, but less frequent use of nonsteroidal antiinflammatory drugs (NSAIDs) among community dwellers with cognitive disorders compared to other older adults [11, 12]. Reports on opioid use have been more inconsistent, but a higher prevalence of opioid use, strong opioid use, and long-term opioid use have also been found among persons with cognitive disorders compared to those without [12-16].

Previous studies have also reported less opioid use after a hip fracture in hospital care among persons with dementia compared to other hip-fracture patients [8, 15, 17-19]. In the days following the fracture, opioids have been used by more than $70 \%$ of people with and without dementia [15], but by fewer and at lower doses in those with dementia $[8,15,17]$. However, among older adults with a hip fracture, pain is still experienced by $42 \%$ until 4 months and by $13-25 \%$ until 1 year [20,21]. Among the population in general, opioid use can be frequent even a year after hip fracture surgery [22]. To our knowledge, there are no previous studies describing the use of opioids after discharge to community settings among hip-fracture patients with cognitive disorders.

\subsection{Aim of the Study}

The aim of this study was to investigate the prevalence of opioid use in the 6 months after discharge from hospital care due to hip fracture among community-dwelling persons with and without $\mathrm{AD}$.

\subsection{Ethics Approval}

All data from the registers were pseudonymized prior to being submitted to the research group. Participants were not contacted in any way, and according to Finnish legislation, ethics committee approval was therefore not required. The study protocol was approved by the register maintainers [the Social Insurance Institution of Finland (SII), Statistics Finland, and the National Institute of Health and Welfare].

\section{Method}

This study is part of the nationwide, register-based Medication use and Alzheimer's disease (MEDALZ) cohort. The cohort contains all community-dwelling persons who received a clinically verified diagnosis of AD during 2005-2011 in Finland $(N=70,718)$. For each person with $\mathrm{AD}$, a comparison person without $\mathrm{AD}$ was matched on age, sex, and region of residence at the time of AD diagnosis $(N=70,718)$. The MEDALZ cohort has been described in more detail elsewhere [23].

The MEDALZ cohort contains data from several nationwide healthcare registers [the Prescription Register (1995-2015), Special Reimbursement Register (1972-2015), and Hospital Discharge Register (1972-2015)] and data on socioeconomic position from Statistics Finland [24-26]. Persons with AD were identified from the Special Reimbursement Register maintained by the SII. The register contains data on all persons who are eligible for special reimbursements for drugs for chronic illnesses, including AD. Reimbursement for antidementia drugs requires an $\mathrm{AD}$ diagnosis based on the National Institute of Neurological and Communicative Diseases and Stroke/Alzheimer's Disease and Related Disorders Association (NINCDS-ADRDA) [27] and Diagnostic and Statistical Manual of Mental Disorders 4th edition (DSM-IV) criteria [28], including a computed tomography or magnetic resonance imaging scan, and confirmation of the diagnosis by a neurologist or a geriatrician. A certificate of the fulfillment of the diagnostic criteria is submitted to the SII, where an expert in this field grants the special reimbursement if the criteria are met. Finnish national guidelines recommend treating all persons with $\mathrm{AD}$ with antidementia drugs unless specifically contraindicated [29]. Reimbursement is granted to persons with mildto-moderate $\mathrm{AD}$, but is not withdrawn when the disease progresses.

For this study, persons having a hip fracture after AD diagnoses/corresponding matching date between January 1, 2011 and until June 30, 2015 were identified. We collected data on hip fractures from the national Hospital Discharge Register, based on the International Classification of Diseases (ICD), with the following ICD-10 codes: S72.0 (fracture neck of femur), S72.1 (peritrochanteric fracture), and S72.2 (subtrochanteric fracture). Persons with a previous hip fracture were identified with the corresponding ICD- 8 and ICD-9 codes, and they were excluded to restrict analyses to incident cases. The positive predictive value (PPV) for $\mathrm{AD}$ 
diagnoses is 97.1 [30], and the PPV for hip fracture is 98 [31].

As the purpose was to investigate opioid use after discharge from hospital care, persons who died during continuous hospital care or within 3 days after discharge were excluded. To ensure follow-up for potential opioid use in outpatient care, further exclusions were made: we excluded persons who were still in hospital care (any hospital care, including acute care hospitals and community hospitals providing rehabilitation) 120 days after hip fracture, those who had less than 10 days of follow-up after discharge, and those who did not purchase any drugs within 60 days after discharge, which was considered as an indicator of institutionalization. Exclusions are described in detail in Fig. 1.
The follow-up in this study was divided into two parts. A pre-fracture period covered 6 months before a hip fracture and a post-discharge period covered 6 months after discharge from hospital care after a hip fracture (Fig. 2a). Between these pre-fracture and post-discharge periods was time spent in hospital care (including surgical care and rehabilitation), during which drug use is not recorded in the Prescription Register and, thus, cannot be assessed in this study. The follow-up in the post-discharge period ended on death, end of the follow-up (December 31, 2015), and, for comparison persons, if they were diagnosed with $\mathrm{AD}$, whichever came first.

Drug use was obtained from the Prescription Register, which includes data on all reimbursed prescription drug purchases from Finnish pharmacies. In Finland, opioids

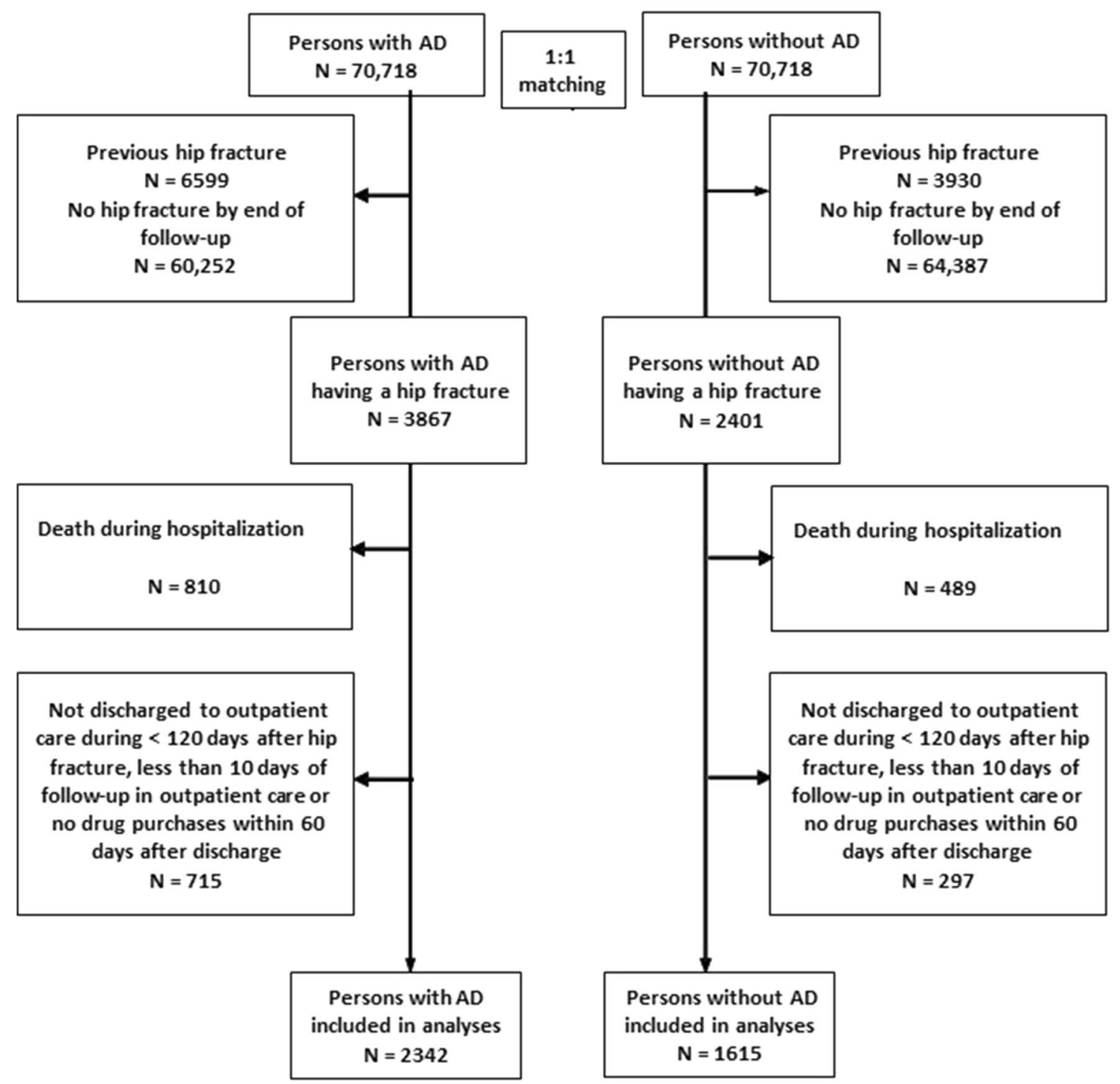

Fig. 1 The formation of the study sample. Persons having a hip fracture after AD diagnoses/corresponding matching date, between January 1, 2011 and until June 30, 2015, were identified. $A D$ Alzheimer's disease 


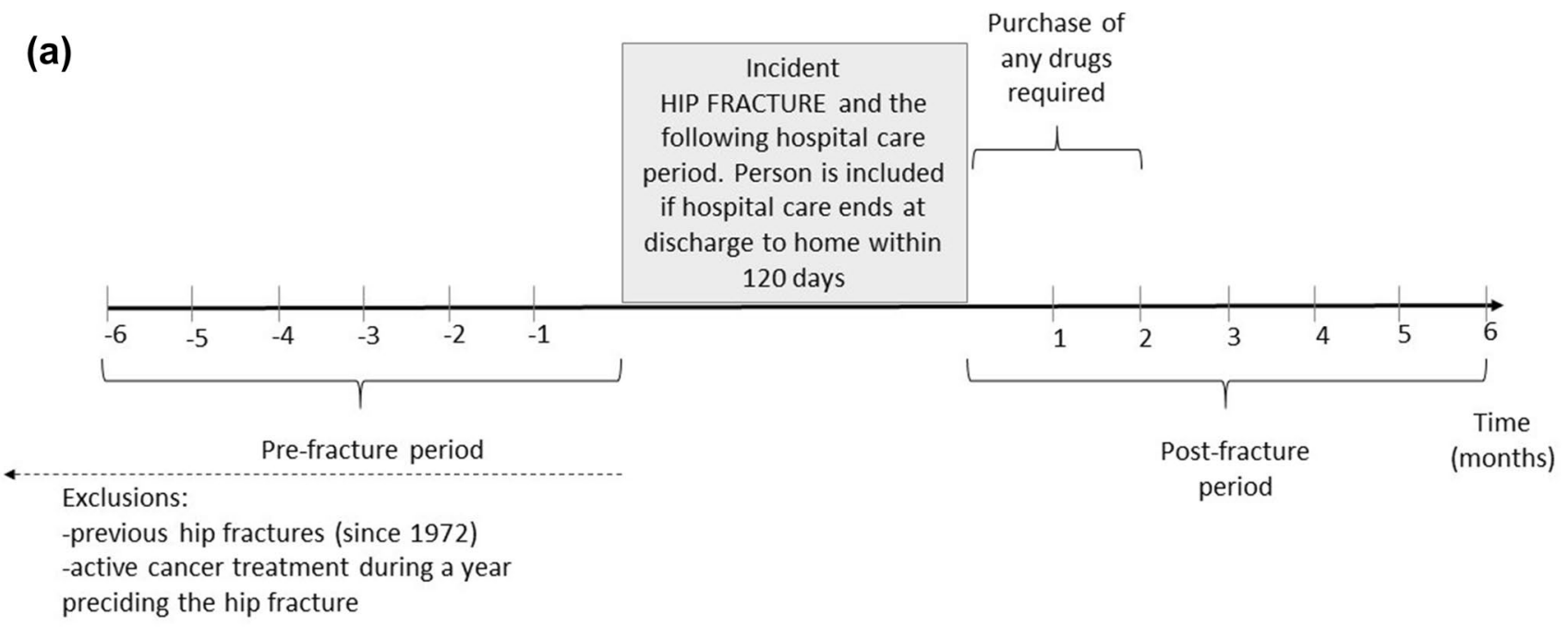

(b) EXAMPLES OF PREVALENCE ASSESSMENT WITH MODELLED DRUG USE PERIODS

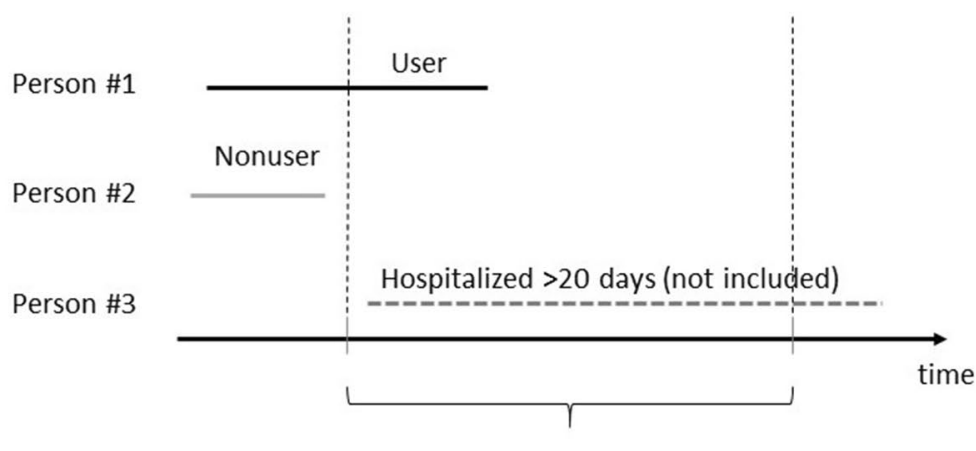

Time window of 30 days

Prevalence $(\%)=\frac{\text { Users }}{\text { Users }+ \text { nonusers }} \times 100$

Fig. 2 a Study design graph. b Definition of opioid use within a 30 -day time window. Opioid users were required to have $\geq 1$ day of opioid use in the time window, according to modeled drug use peri-

are only available with prescription and opioids are reimbursed, with few exceptions. Products containing paracetamol and codeine were not reimbursed prior to June 2010, which is why we restricted our analyses only on hip fractures from 2011 onward. Drugs used during hospital stays are not recorded in this register and were not analyzed in this study. Drugs in the Prescription Register were categorized according to the World Health Organization (WHO) Anatomical Therapeutic Chemical (ATC) classification system [32]. Opioids (N02A) were categorized into weak opioids [tramadol (N02AX02) and codeine (N02AA59)], partial opioid agonists [buprenorphine (N02AE01)], and strong opioids [oxycodone (N02AA05), fentanyl (N02AB03), morphine (N02AA01), hydromorphone (N02AA03), ods. Opioid use was only assessed among those who had $\geq 10$ days of follow-up within the 30-day window, due to hospital care periods and mortality

dextropropoxyphene (N02AC04), and pentazocine (N02AD01)]. Drug use periods for each opioid were modeled with a previously validated mathematical method, 'from purchases to drug use periods', PRE2DUP [33, 34]. The method calculates drug use periods based on sliding averages of daily dose in defined daily doses (DDDs) and according to individual drug use patterns. It takes into account time spent in hospital care (when drugs are provided by the caring unit and not recorded in the register), stockpiling of drugs, and changing dose during use.

Opioid use prevalence was investigated during the prefracture period and the post-discharge period. Opioid use was assessed in 30-day time windows as 'used' (at least 1 day) versus 'not used,' based on modeled drug use data 
(Fig. 2b). Opioid use was only assessed among those who had at least 10 days of follow-up in the time window (i.e., censoring those hospitalized for more than 20 days of the period and excluding those who had died). Thus, the sample size varies in each time window. For the postdischarge period, also the proportion of those who used opioids at any time point during the period was calculated separately.

The analyses on factors associated with opioid use after hip fracture were adjusted for covariates associated with opioid use and hip fracture (Supplementary Table 1; see the electronic supplementary material). The Special Reimbursement Register and Hospital Discharge Register were utilized to identify asthma or chronic obstructive pulmonary disease (COPD), any cardiovascular disease, active cancer, diabetes (type 1 and type 2), and rheumatoid arthritis and other connective tissue diseases, until the date of hip fracture. Any cardiovascular disease was defined as one or more of the following: arterial hypertension, coronary artery disease, chronic heart failure, or chronic arrhythmia. Prosthetic replacement of knee or hip was identified from the Hospital Discharge Register with the Finnish version of the Nordic Medico-Statistical Committee (NOMESCO) Classification for Surgical Procedures (NCSP). Bisphosphonate use (M05BA, M05BB), from the Prescription Register, was utilized as a proxy for osteoporosis and measured as ever used before the hip fracture.

The Prescription Register was utilized to identify the use of other drugs during the month before hip fracture: non-opioid analgesics, antiepileptics, and psychotropics. Psychotropic drugs were defined as benzodiazepines and related drugs (N05BA, N05CD, and N05CF), antidepressants (N06A), and antipsychotics (N05A, excluding lithium). Of non-opioid analgesics, paracetamol, ibuprofen, and ketoprofen are also available as over-the-counter products in Finland in small package sizes, which are not recorded in the Prescription Register.

Socioeconomic position was defined as the highest occupational position recorded in population censuses for study persons in their middle age (45-55 years old). Socioeconomic position was based on classification by Statistics Finland, and it was categorized into four classes: the highest class included entrepreneurs and higher clerical workers; the medium class included lower clerical workers and employees; and the lowest class included unemployed persons, retirees, and students. Persons with unknown socioeconomic class and those with missing data at Statistics Finland (1.9\% of the cohort) were combined in the class 'unknown.'

All statistical analyses were performed using SPSS (version 24.0; IBM Corp., Armonk, New York, USA). Characteristics between persons with and without AD were compared with chi-squared tests for categorical variables. We considered $P \leq 0.05$ as statistically significant. The median, with interquartile range (IQR), was calculated for continuous variables.

Logistic regression models were used to investigate factors associated with the use of opioids, weak opioids, buprenorphine, and strong opioids in the post-discharge period (Supplementary Table 2; see the electronic supplementary material). The results were expressed as odds ratios (ORs) with $95 \%$ confidence intervals (CIs). The analyses were adjusted for the following factors: $\mathrm{AD}$, sex, age $(<80$ vs. $\geq 80$ years), time since $\mathrm{AD}$ diagnosis (categorized as 0 to $<3,3$ to $<6,6$ to $<9$, and 9 years or more), socioeconomic position (high, medium, low, or unknown), asthma/COPD, rheumatoid arthritis, diabetes, history of stroke, active cancer, epilepsy, cardiovascular disease, endoprosthesis, osteoporosis, history of fracture, substance abuse, use of psychotropics, antiepileptics or non-opioid analgesics, and the length of hospital stay (categorized as 0-7, 8-14, 15-21, $22-28,29-35$, or 36 days or more). In the sensitivity analyses, we investigated opioid use after hospital care among persons with and without $\mathrm{AD}$ who were discharged after a hip fracture in 0-21 days and 22-120 days to further analyze the effect of length of stay on opioid use.

\section{Results}

In this study, there were 2342 persons with and 1615 persons without AD who had a new hip fracture. The mean age was 84 years [standard deviation (SD) 6.4 years] for persons with $\mathrm{AD}$ and 87 years (SD 5.1 years) for persons without AD. Women comprised $79 \%$ of persons with $\mathrm{AD}$ and $80 \%$ of persons without $\mathrm{AD}$. The median time from the AD diagnoses/corresponding matching date for comparison persons to having a hip fracture was 1365 days (IQR 814-1950) among persons with AD and 1554 days (IQR 963-2225) among persons without AD. After the discharge from hospital care due to hip fracture, 506 persons (12.7\%) died within the first 6 months after discharge: 341 persons with $\mathrm{AD}$ and 165 persons without $\mathrm{AD}$.

Opioid use was more frequent among persons with $\mathrm{AD}$, with $39.5 \%$ (95\% CI 37.5-41.5) using after hip fracture in comparison to $31.2 \%$ (95\% CI 28.9-33.5) (Table 1). Opioid users were more likely to have $\mathrm{AD}$, cardiovascular disease, history of fracture, and osteoporosis and were in a lower socioeconomic position. In addition, opioid users more often used psychotropics, antiepileptics, and non-opioid analgesics in the pre-fracture period. Nonusers more likely had hip or knee endoprosthesis and were more likely in a high socioeconomic position. Among persons with $\mathrm{AD}$, the proportion of opioid users after hip fracture became larger in later annual cohorts compared to earlier cohorts. In 2011, $32.7 \%$ of persons with $\mathrm{AD}$ were opioid users compared to 
Table 1 Characteristics of users and nonusers of opioids in a 6-month post-fracture period among persons with and without AD

\begin{tabular}{|c|c|c|c|c|c|c|}
\hline \multirow[t]{2}{*}{ Female sex } & \multicolumn{3}{|l|}{ Without AD } & \multicolumn{2}{|l|}{ With AD } & \multirow[t]{2}{*}{$P$ value } \\
\hline & $\begin{array}{l}\text { Opioid user } \\
N=503 \\
N(\%)\end{array}$ & $\begin{array}{l}\text { Nonuser } \\
N=1112 \\
N(\%)\end{array}$ & $P$ value & $\begin{array}{l}\text { Opioid user } \\
N=925 \\
N(\%)\end{array}$ & $\begin{array}{l}\text { Nonuser } \\
N=1417 \\
N(\%)\end{array}$ & \\
\hline Female sex & $403(80.1)$ & $881(79.2)$ & 0.681 & $746(80.6)$ & $1102(77.8)$ & 0.095 \\
\hline Age, years & & & 0.553 & & & 0.078 \\
\hline$<65$ & 0 & 0 & & $9(1.0)$ & $11(0.8)$ & \\
\hline $65-74$ & $12(2.4)$ & $18(1.6)$ & & $54(5.8)$ & $109(7.7)$ & \\
\hline $75-84$ & $168(33.4)$ & $367(33.0)$ & & $363(39.2)$ & $600(42.3)$ & \\
\hline$\leq 85$ & $323(64.2)$ & $727(65.4)$ & & 499 (53.9) & $697(49.2)$ & \\
\hline Socioeconomic position & & & 0.214 & & & 0.056 \\
\hline High & $152(30.2)$ & $395(35.5)$ & & $284(30.7)$ & $488(34.4)$ & \\
\hline Medium & $310(61.6)$ & $635(57.1)$ & & $563(60.9)$ & $831(58.6)$ & \\
\hline Low & $33(6.6)$ & $68(6.1)$ & & $63(6.8)$ & $88(6.2)$ & \\
\hline Unknown & $8(1.6)$ & $14(1.3)$ & & $15(1.6)$ & $10(0.7)$ & \\
\hline Asthma/COPD & $64(12.7)$ & $121(10.9)$ & 0.282 & $105(11.4)$ & $136(9.6)$ & 0.172 \\
\hline Rheumatoid arthritis & $44(8.7)$ & $73(6.6)$ & 0.117 & $56(6.1)$ & $67(4.7)$ & 0.160 \\
\hline Diabetes & $112(22.3)$ & $206(18.5)$ & 0.080 & $144(15.6)$ & $283(20.0)$ & 0.007 \\
\hline Cardiovascular disease & 397 (78.9) & $793(71.3)$ & 0.001 & $629(68.0)$ & $916(64.6)$ & 0.094 \\
\hline Previous stroke & $66(13.1)$ & $152(13.7)$ & 0.765 & $114(12.3)$ & $145(10.2)$ & 0.115 \\
\hline Epilepsy & $14(2.8)$ & $28(2.5)$ & 0.756 & $29(3.1)$ & $34(2.4)$ & 0.282 \\
\hline Endoprosthesis* & $270(53.7)$ & $599(53.9)$ & 0.944 & $461(49.8)$ & $820(57.9)$ & $<0.001$ \\
\hline History of fracture & $164(32.6)$ & $344(30.9)$ & 0.504 & $347(37.5)$ & $464(32.7)$ & 0.018 \\
\hline Osteoporosis & $144(28.6)$ & $211(19.0)$ & $<0.001$ & $203(21.9)$ & $222(15.7)$ & $<0.001$ \\
\hline Active cancer & $28(5.6)$ & $55(4.9)$ & 0.601 & $53(5.7)$ & $59(4.2)$ & 0.083 \\
\hline History of substance abuse & $13(2.6)$ & $21(1.9)$ & 0.367 & $24(2.6)$ & $36(2.5)$ & 0.936 \\
\hline \multicolumn{7}{|l|}{ Use of drugs** } \\
\hline Psychotropics & $226(44.9)$ & $391(35.2)$ & $<0.001$ & $598(64.6)$ & $833(58.8)$ & 0.004 \\
\hline Antiepileptics & $54(10.7)$ & $61(5.5)$ & $<0.001$ & $78(8.4)$ & $79(5.6)$ & 0.007 \\
\hline Non-opioid analgesics & $243(48.3)$ & $335(30.1)$ & $<0.001$ & $454(49.1)$ & $462(32.6)$ & $<0.001$ \\
\hline Length of hospital stay & & & 0.399 & & & $<0.001$ \\
\hline $0-7$ days & $54(10.7)$ & $98(8.8)$ & & $211(22.8)$ & $177(12.5)$ & \\
\hline 8-14 days & $62(12.3)$ & $137(12.3)$ & & $152(16.4)$ & $169(11.9)$ & \\
\hline 15-21 days & $86(17.1)$ & $169(15.2)$ & & $166(17.9)$ & $252(17.8)$ & \\
\hline 22-28 days & $86(17.1)$ & $171(15.4)$ & & $117(12.6)$ & $205(14.5)$ & \\
\hline 29-35 days & $51(10.1)$ & $123(11.1)$ & & $82(8.9)$ & $150(10.6)$ & \\
\hline 36 days or more & $164(32.6)$ & $414(37.2)$ & & $197(21.3)$ & $464(32.7)$ & \\
\hline Year of hip fracture & & & 0.722 & & & $<0.001$ \\
\hline 2011 & $99(19.7)$ & $253(22.8)$ & & $181(19.6)$ & $372(26.3)$ & \\
\hline 2012 & $124(24.7)$ & $264(23.7)$ & & $217(23.5)$ & $365(25.8)$ & \\
\hline 2013 & 109 (21.7) & $240(21.6)$ & & $243(26.3)$ & $305(21.5)$ & \\
\hline 2014 & $118(23.5)$ & $245(22.0)$ & & $187(20.2)$ & $267(18.8)$ & \\
\hline 2015 & $53(10.5)$ & $110(9.9)$ & & $97(10.5)$ & $108(7.6)$ & \\
\hline
\end{tabular}

$A D$ Alzheimer's disease, $C O P D$ chronic obstructive pulmonary disease

*Of knee or hip joint

**Last 0-30 days in pre-fracture period

47.3\% in 2015. Among persons without AD, the increase in proportion was smaller, from $28.1 \%$ in 2011 to $32.5 \%$ in $2015(P=0.254)$.
In the pre-fracture period, there were no significant differences in opioid use between persons with $\mathrm{AD}$ and those without AD. During the post-discharge period, persons 
with $\mathrm{AD}$ used opioids more often than persons without $\mathrm{AD}$ (Fig. 3a). Weak opioids were more likely used by persons without $\mathrm{AD}$ after the first month in the post-discharge period (Fig. 3b), whereas buprenorphine was more frequently used by persons with $\mathrm{AD}$ during the entire post-discharge period (Fig. 3c). Strong opioid use was more frequent among persons with $\mathrm{AD}$ during the first 3 months of the post-discharge period (Fig. 3d). Of the persons who used any opioids in the post-discharge period, $37.2 \%$ of persons with $\mathrm{AD}$ and $44.7 \%$ without $\mathrm{AD}$ had used opioids in the pre-fracture period.

In sensitivity analyses on the effect of length of hospital stay, we compared those discharged after a hip fracture in 0-21 days with those staying in the hospital for 22-120 days. Of the persons who were discharged in 0-21 days, 46.9\% (95\% CI 44.0-49.9) of persons with AD and 33.3\% (95\% CI 29.7-37.2) without $\mathrm{AD}$ used opioids in the post-discharge period. Of the persons who were discharged in 22-120 days, $32.6 \%$ (95\% CI 30.0-35.3) of persons with $\mathrm{AD}$ and $29.8 \%$ (95\% CI 27.1-32.7) of persons without AD used opioids in the post-discharge period.

$\mathrm{AD}$ was associated with the use of buprenorphine [adjusted OR (aOR) 2.02, 95\% CI 1.67-2.45] and strong opioids (aOR 1.70, 95\% CI 1.38-2.09), but inversely associated with the use of weak opioids (aOR $0.56,95 \%$ CI $0.46-0.69$ ) (see Supplementary Table 2).

\section{Discussion and Conclusion}

Our main finding was that persons with $\mathrm{AD}$ frequently used opioids after discharge from hospital care due to hip fracture. Strong opioid use during the first 3 months and buprenorphine use throughout the post-discharge period were significantly more frequent in persons with $\mathrm{AD}$, whereas weak opioids were significantly less frequently used in the $\mathrm{AD}$ group, compared to persons without AD. To our knowledge, this is the first study to investigate the use of opioids after discharge from hospital care after hip fracture among persons with a verified diagnosis of AD.

Paracetamol is recommended as a first-line analgesic after discharge from hospital after hip fracture [29], and opioid use in older adults should ideally be reserved for management of severe acute pain [35]. Decisions to continue treatment with opioids are done based on individual assessment of pain. However, opioid use can still be common 1 year after hip fracture surgery [22]. Pain assessment is essential in monitoring pain treatment and for decisions to continue, taper, or discontinue opioid therapy [36-38]. Among persons with cognitive disorders, however, assessment of pain can be challenging [5]. Self-report is important in mild-tomoderate $\mathrm{AD}$, but observational pain scales, such as Pain Assessment in Advanced Dementia (PAINAD) and Pain Assessment Checklist for Seniors with Limited Ability to
Communicate (PACSLAC) [39, 40], become more useful as the disease progresses [5, 41, 42]. Frequent use of opioids months after the hip fracture among persons with $A D$ in this study may indicate the challenges of pain assessment. However, whether regular pain assessment has been carried out could not be evaluated in this study. Similarly, indication for opioid use could not be assessed through the registers utilized in this study. Thus, it is possible that some persons used opioids for pain unrelated to the hip fracture.

Studies on the use of opioids after hospital care for hip fractures are rare. Previous studies have reported the use of opioids among persons with $\mathrm{AD}$ in hospital care or in nursing homes after a hip fracture, and have described possible undertreatment of pain among persons with dementia $[8,15$, $17,18]$. Persons with dementia who had had a hip fracture received less treatment with opioids in hospital care $[8,15$, $17,18]$ and less treatment with paracetamol and opioids [15] compared to persons without dementia. In contrast, we found more frequent use of buprenorphine and strong opioids among persons with $\mathrm{AD}$. While it is possible that differences exist between prescribing patterns of different countries, the contrast between previous studies and ours is likely to reflect the difference in treatment setting, i.e., between inpatient hospital care and the community. Persons with $\mathrm{AD}$ who are discharged to community settings might thus benefit from closer monitoring of their opioid treatment. In our study, persons with $\mathrm{AD}$ also had shorter hospital stays compared to persons without $\mathrm{AD}$, which is in line with previous research [43]. However, adjusting for length of stay did not change our results. In our sensitivity analyses stratified by length of hospital stay, persons with AD still used significantly more opioids than those without AD. One possible explanation for this finding is that a larger proportion of persons with $\mathrm{AD}$ are clients of residential and home care and so their pain is attended to more frequently. In addition, research in recent decades may have increased attention to pain management in persons with dementia. This may be reflected in previous findings of similar or even higher prevalence rates of analgesic and opioid use among community dwellers with dementia, when compared to other older adults [12-16]. Moreover, we found that among persons with $\mathrm{AD}$, the proportion of opioid users after hip fracture increased steadily by almost 15 percentage points from 2011 to 2015. Increased use of opioids among persons with cognitive disorders has been previously reported among community-dwelling Finns, as well as in Finnish, Norwegian, and Canadian long-term care facilities [12, 44-46]. Whether these trends persist over time should be a subject for future studies.

In our study population, buprenorphine was almost solely used as transdermal patches. We found that buprenorphine use was significantly more frequent in persons with $\mathrm{AD}$ throughout the post-discharge period, which is in accordance with previous findings where studies have described 
Fig. 3 Prevalence of opioid use among persons with and without $\mathrm{AD}$ in pre-fracture and post-discharge periods: a any opioids, b weak opioids, c buprenorphine, and $\mathbf{d}$ strong opioids. $A D$ Alzheimer's disease
(\%)

(a) Any opioids

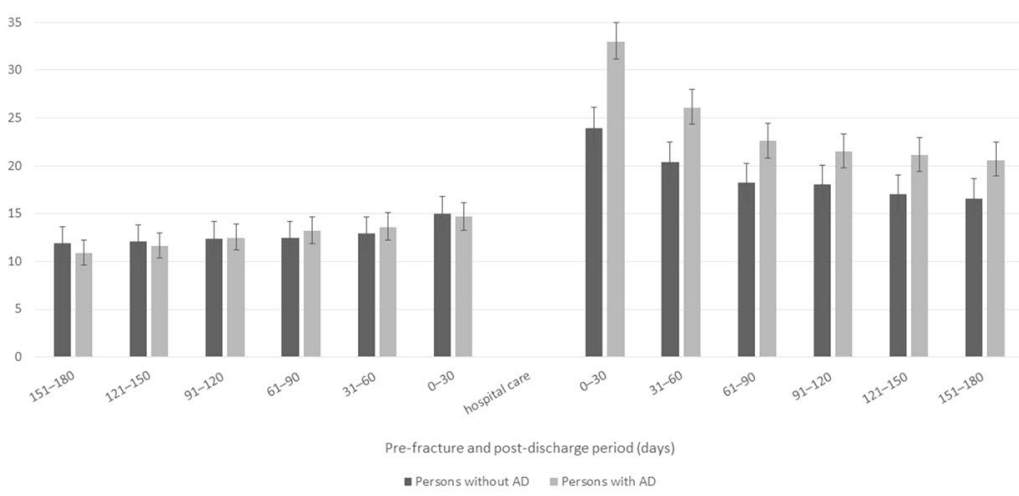

(\%)

(b) Weak opioids

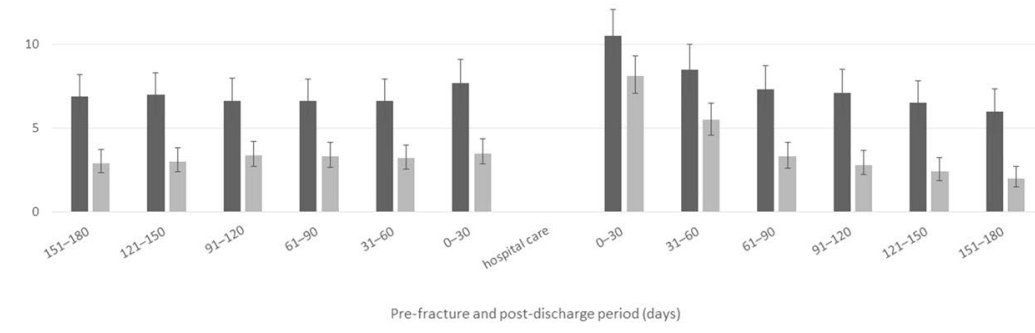

(\%)

(c) Buprenorphine

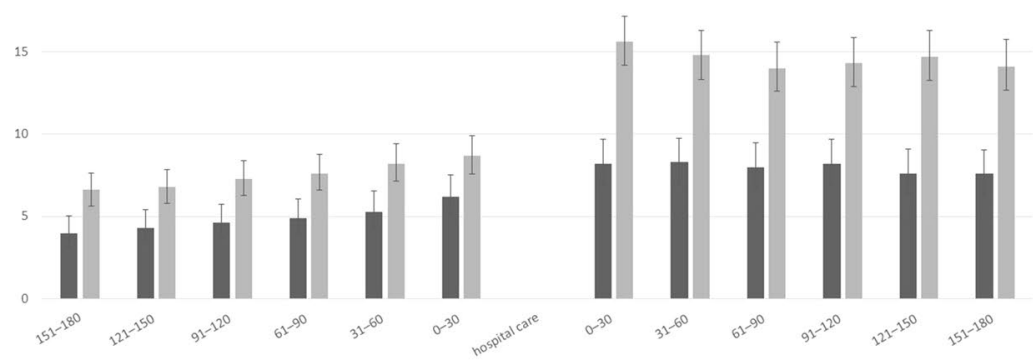

Pre-fracture and post-discharge period (days)

- Persons without $A D=$ Persons with $A D$

(\%)

(d) Strong opioids

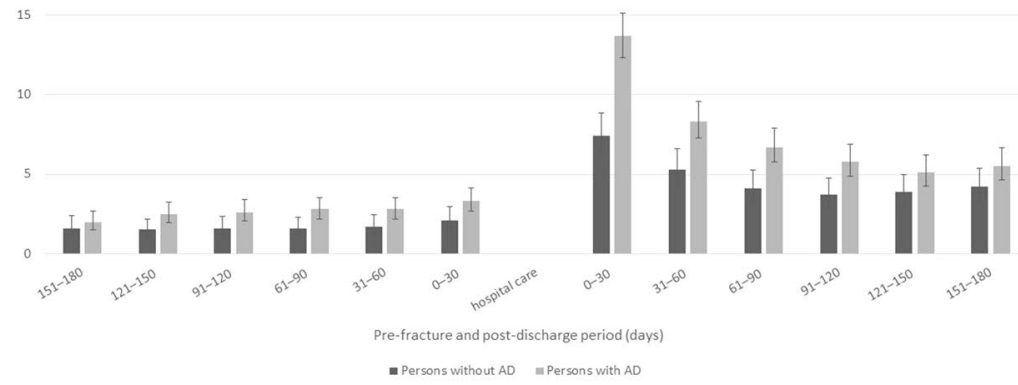


longer persistence on transdermal opioids compared with oral ones $[47,48]$ and more frequent use of transdermal opioids among community-dwellers with dementia [12, 20]. In addition, long-term opioid use has been strongly associated with transdermal opioid use among persons with AD [14]. Similarly, dementia has previously been reported as a risk factor for chronic opioid use after total hip arthroplasty [49]. Transdermal opioid treatment may be preferred due to difficulties in the ingestion of oral medicines, and particularly in patients with moderate to severe AD [50]. Treatment schemes of transdermal buprenorphine, i.e., change every 7 days, may provide additional ease of use, for example, reducing administration time and staffing requirements in home care services and in residential care [51]. In addition, it may not be surprising that buprenorphine is preferred over weak opioids among persons with AD; weak opioids may commonly induce constipation and drug-drug interactions, and have genetic variation in efficacy $[34,52]$. Use of tramadol may be problematic among older people, as it can cause central nervous system adverse effects, and thus, tramadol is recommended to be avoided in older adults [33]. Further, antidepressant use, which may increase the serotonergic adverse effects of tramadol, is common among persons with AD [53]. However, clinicians should be aware that buprenorphine adverse effects, such as somnolence, may be more pronounced among concomitant antidepressant users with dementia [54, 55]. Regular assessment of pain and the need for opioids, as well as the benefits and harms of opioid therapy, should be conducted, regardless of the route of administration.

The main strength of our study was the nationwide coverage of all community-dwelling persons with a clinically verified $\mathrm{AD}$ diagnosis from several years. Our results are generalizable to prescription opioid use and to older persons with and without $\mathrm{AD}$, due to the nationwide registers with good coverage and validity [56], although some differences between countries may exist. In this study, the diagnosis of hip fracture is reliably recorded in the Hospital Discharge Register data [31]. The diagnosis of AD cases can be considered reliable due to the diagnosis requirements of the SII [30]. However, mixed cases of AD with features of vascular or Lewy body dementia may be included if AD was considered the main contributor to the symptoms. In addition to previously mentioned strengths, our register-based results are not prone to recall bias, which can be a problem in interview-based studies.

A limitation of this study was that we were not able to analyze opioid usage in different stages of AD because the Special Reimbursement register does not include the severity of the disease. The registers do not contain data on pain severity or non-pharmacological treatments for pain, and thus, it is not possible to assess the adequacy of the provided pharmacotherapy. Moreover, we were not able to estimate opioid dose during each 30-day time window, and this is therefore an important topic for future research. In addition, we were not able to analyze opioid use during hospital care or if persons were discharged to institutions which provide medications, i.e., are not recorded in the Prescription Register. However, persons without outpatient follow-up and those who did not purchase any drugs within 3 months following discharge were not included in our study. The results of our study may at least partly be explained by country-specific prescribing patterns, and should thus be replicated in other countries.

Persons with AD more frequently used opioids than persons without $\mathrm{AD}$ after hospital discharge after hip fracture, and thus, our study could not confirm low or undertreatment of pain after hospitalization due to hip fracture in persons with AD. Frequent opioid use after hospital care due to hip fracture may indicate increased attention to pain among persons with AD. Nevertheless, pain and the benefits and harms of opioid use should be evaluated regularly in community-dwelling older persons after hospitalization due to hip fracture.

Acknowledgements Open access funding provided by University of Eastern Finland (UEF) including Kuopio University Hospital.

Author Contributions All authors contributed to the study conception and design. Data preparation and analysis were performed by Eveliina Lintula and Heidi Taipale. The first draft of the manuscript was written by Eveliina Lintula, and all authors read and approved the final manuscript and commented on previous versions of the manuscript.

\section{Compliance with Ethical Standards}

Funding This research did not receive any funding from agencies in the public, commercial, or not-for-profit sectors

Conflict of Interest HT, JT, and AT have participated in research projects funded by Janssen and Eli Lilly, with grants paid to the institution where they were employed. JT has received personal fees from the Finnish Medicines Agency (Fimea), European Medicines Agency (EMA), Eli Lilly, Janssen-Cilag, Lundbeck, and Otsuka, and has received grants from the Stanley Foundation and Sigrid Jusélius Foundation. SH has received fees from the Swedish Research Council. AH has been supported by the Alfred Kordelin Foundation and Borg Foundation. All other authors (EL, MT, and AMT) declare no conflicts of interest.

Ethical Approval Participants were not contacted in any way, and according to Finnish legislation, ethics committee approval was therefore not required.

Open Access This article is licensed under a Creative Commons Attribution-NonCommercial 4.0 International License, which permits any non-commercial use, sharing, adaptation, distribution and reproduction in any medium or format, as long as you give appropriate credit to the original author(s) and the source, provide a link to the Creative Commons licence, and indicate if changes were made. The images or other third party material in this article are included in the article's Creative 
Commons licence, unless indicated otherwise in a credit line to the material. If material is not included in the article's Creative Commons licence and your intended use is not permitted by statutory regulation or exceeds the permitted use, you will need to obtain permission directly from the copyright holder. To view a copy of this licence, visit http://creativecommons.org/licenses/by-nc/4.0/.

\section{References}

1. Alzheimer's Association. Alzheimer's disease facts and figures. Alzheimers Dement. 2018;2018(3):367-429.

2. Baker NL, Cook MN, Arrighi HM, Bullock R. Hip fracture risk and subsequent mortality among Alzheimer's disease patients in the United Kingdom, 1988-2007. Age Ageing. 2011;40:49-54.

3. Tolppanen AM, Lavikainen P, Soininen H, Hartikainen S. Incident hip fractures among community dwelling persons with Alzheimer's disease in a Finnish nationwide register-based cohort. PLoS One. 2013;8:e59124.

4. Hadjistavropoulos T, Herr K, Prkachin KM, Craig KD, Gibson SJ, Lukas A, Smith JH. Pain assessment in elderly adults with dementia. Lancet Neurol. 2014;13(12):1216-27.

5. Corbett A, Husebo B, Malcangio M, Staniland A, Cohen-Mansfield J, Aarsland D, Ballard C. Assessment and treatment of pain in people with dementia. Nat Rev Neurol. 2010;8:264-74.

6. Feast AR, White N, Lord K, Kupeli N, Vickerstaff V, Sampson EL. Pain and delirium in people with dementia in the acute general hospital setting. Age Ageing. 2018;47:841-6.

7. Husebo BS, Strand LI, Moe-Nilssen R, Borgehusebo S, Aarsland D, Ljunggren AE. Who suffers most? Dementia and pain in nursing home patients: a cross-sectional study. J Am Med Dir Assoc. 2008;9:427-33.

8. Morrison RS, Siu AL. A comparison of pain and its treatment in advanced dementia and cognitively intact patients with hip fracture. J Pain Symptom Manage. 2000;19:240-8.

9. Nygaard HA, Jarland M. Are nursing home patients with dementia diagnosis at increased risk for inadequate pain treatment? Int J Geriatr Psych. 2005;20:730-7.

10. Shega JW, Hougham GW, Stocking CB, Cox-Hayley D, Sachs GA. Pain in community-dwelling persons with dementia: frequency, intensity, and congruence between patient and caregiver report. J Pain Symptom Manage. 2004;28:585-92.

11. Haasum Y, Fastbom J, Fratiglioni L, Kåreholt I, Johnell K. Pain treatment in elderly persons with and without dementia: a population-based study of institutionalized and home-dwelling elderly. Drugs Aging. 2011;28:283-93.

12. Hamina A, Taipale H, Tanskanen A, Tolppanen AM, Tiihonen J, Hartikainen S. Differences in analgesic use in communitydwelling persons with and without Alzheimer's disease. Eur J Pain. 2017;21:658-67.

13. Bell JS, Laitinen ML, Lavikainen P, Lönnroos E, Uosukainen $\mathrm{H}$, Hartikainen S. Use of strong opioids among communitydwelling persons with and without Alzheimer's disease in Finland. Pain. 2011;152:543-7.

14. Hamina A, Taipale H, Tanskanen A, Tolppanen AM, Karttunen N, Pylkkönen L, Tiihonen J, Hartikainen S. Long-term use of opioids for nonmalignant pain among community-dwelling persons with and without Alzheimer disease in Finland: a nationwide register-based study. Pain. 2017;158:252-60.

15. Jensen-Dahm C, Palm H, Gasse C, Dahl JB, Waldemar G. Postoperative treatment of pain after hip fracture in elderly patients with dementia. Dement Geriatr Cogn Disord. 2016;41:181-91.

16. Shen C, Zhao X, Dwibedi N, Wiener RC, Findley PA, Sambamoorthi U. Opioid use and the presence of Alzheimer's disease and related dementias among elderly Medicare beneficiaries diagnosed with chronic pain conditions. Alzheimer's Dementia Transl Res Clin Intervent. 2018;4:661-8.

17. Adunsky A, Levy R, Mizrahi E, Arad M. Exposure to opioid analgesia in cognitively impaired and delirious elderly hip fracture patients. Arch Gerontol Geriatr. 2002;35:245-51.

18. Feldt KS, Gunderson J. Treatment of pain for older hip fracture patients across settings. Orthop Nurs. 2002;21(5):66-71.

19. Sieber FE, Mears S, Lee H, Gottschalk A. Postoperative opioid consumption and its relationship to cognitive function in older adults with hip fracture. J Am Geriatr Soc. 2011;59:2256-62.

20. Dasch B, Endres HG, Maier C, Lungenhausen M, Smektala R, Trampisch HJ, Pientka L. Fracture-related hip pain in elderly patients with proximal femoral fracture after discharge from stationary treatment. Eur J Pain. 2008;12(2):149-56.

21. Dimitriou R, Calori GM, Giannoudis PV. Improving patients' outcomes after osteoporotic fractures. Int J Clin Rheumatol. 2012;7(1):109-24.

22. Simoni AH, Nikolajsen L, Olesen AE, Christiansen CF, Pedersen $\mathrm{AB}$. Opioid use after hip fracture surgery: a Danish nationwide cohort study from 2005-2015. Eur J Pain. 2019. https://doi. org/10.1002/ejp.1392.

23. Tolppanen A-M, Taipale H, Koponen M, Lavikainen P, Tanskanen A, Tiihonen J, Hartikainen S. Cohort profile: the Finnish Medication and Alzheimer's disease (MEDALZ) study. BMJ Open. 2016;6:e 012100.

24. Social Insurance Institution (Kela): Statistics on reimbursements for prescription medicines. https://www.kela.fi/web/en/492.

25. National Institute for Health and Welfare (THL): Care Register for Health Care. https://thl.fi/en/web/thlfi-en/statistics/informatio n-on-statistics/register-descriptions/care-register-for-health-care.

26. Statistics Finland: Classification of socio-economic groups. https ://www.stat.fi/en/luokitukset/sosioekon_asema/.

27. McKhann G, Drachman D, Folstein M, Katzman R, Price D, Stadlan EM. Clinical diagnosis of Alzheimer's disease: report of the NINCDS-ADRDA Work Group under the auspices of Department of Health and Human Services Task Force on Alzheimer's Disease. Neurology. 1984;34:939-44.

28. American Psychiatric Association. Diagnostic and statistical manual of mental disorders. Washington, DC: American Psychiatric Press; 1994.

29. Finnish Medical Society Duodecim. Current care guidelines: hip fracture. 2011. www.kaypahoito.fi. (abstract available in English). Accessed 10 Apr 2019.

30. Solomon A, Ngandu T, Soininen H, Hallikainen MM, Kivipelto M, Laatikainen T. Validity of dementia and Alzheimer disease diagnoses in Finnish national registers. Alzheimers Dement. 2014;10:303-9.

31. Sund R, Nurmi-Lüthje I, Lüthje P, Tanninen S, Narinen A, Keskimäki I. Comparing properties of audit data and routinely collected register data in case of performance assessment of hip fracture treatment in Finland. Methods Inf Med. 2007;5:558-66.

32. WHO Collaborating Center for Drug Statistics Methodology. Norwegian Institute of Public Health. The Anatomical Therapeutic Chemical classification system. http://www.whocc.no/atc_ddd_ index/. Accessed 3 Sep 2018.

33. Taipale H, Tanskanen A, Koponen M, Tolppanen A-M, Tiihonen J, Hartikainen S. Agreement between PRE2DUP register data modeling method and comprehensive drug use interview among older persons. Clin Epidemiol. 2016;8:363-71.

34. Tanskanen A, Taipale H, Koponen M, Tolppanen A-M, Hartikainen S, Ahonen R, Tiihonen J. From prescription drug purchases to drug use periods-a second generation method (PRE2DUP). BMC Med Inform Decis Mak. 2015;15:21. 
35. American Geriatrics Society. American Geriatrics Society 2019 updated AGS Beers criteria ${ }^{\circledR}$ for potentially inappropriate medication use in older adults. J Am Geriatr Soc. 2019;67:674-94.

36. Abdulla A, Adams N, Bone M, Elliott AM, Gaffin J, Jones D, Knaggs R, Martin D, Sampson L, Schofield P. Guidance on the management of pain in older people. Age Ageing. 2013;42(suppl 1):i1-57.

37. Schofield $\mathrm{P}$. The assessment of pain in older people: UK national guidelines. Age Ageing. 2018;48:i1-22.

38. American Geriatrics Society Panel on the Pharmacological Management of Persistent Pain in Older Persons. Pharmacological management of persistent pain in older persons. J Am Ger Soc. 2009; 57:1331-46.

39. Warden V, Hurley AC, Volicer L. Development and psychometric evaluation of the Pain Assessment in Advanced Dementia (PAINAD) scale. J Am Med Dir Assoc. 2003;4:9-15.

40. Fuchs-Lacelle S, Hadjistavropoulos HD. Development and preliminary validation of the Pain Assessment Checklist for Seniors with Limited Ability to Communicate (PACSLAC). Pain Manag Nurs. 2004;1:37-49.

41. Hadjistavropoulos T, Herr K, Prkachin KM, Craig KD, Gibson SJ, Lukas A, Smith JH. Pain assessment in elderly adults with dementia. Lancet Neurol. 2014;13:1216-27.

42. Schofield P, Abdulla A. Pain assessment in the older population: what the literature says. Age Ageing. 2018;47:324-7.

43. Scandol JP, Toson B, Close JC. Fall-related hip fracture hospitalisations and the prevalence of dementia within older people in New South Wales, Australia: an analysis on linked data. Injury. 2013;44:776-83.

44. Roitto HM, Kautiainen H, Aalto UL, Öhman H, Laurila J, Pitkälä $\mathrm{KH}$. Fourteen-year trends in the use of psychotropic medications, opioids, and other sedatives among institutionalized older people in Helsinki, Finland. J Am Med Dir Assoc. 2019;20:305-11.

45. Sandvik R, Selbaek G, Kirkevold O, Aarsland D, Husebo BS. Analgesic prescribing patterns in Norwegian nursing homes from 2000 to 2011: trend analyses of four data samples. Age Ageing. 2016;45:54-60.

46. Iaboni A, Campitelli MA, Bronskill SE, Diong C, Kumar M, Maclagan LC, Gomes T, Tadrous M, Maxwell CJ. Time trends in opioid prescribing among Ontario long-term care residents: a repeated cross-sectional study. CMAJ Open. 2019;7:E582-9.

47. Gallagher AM, Leighton-Scott J, van Staa TP. Utilization characteristics and treatment persistence in patients prescribed low-dose buprenorphine patches in primary care in the United Kingdom: a retrospective cohort study. Clin Ther. 2009;31:1707-15.

48. Lalic S, Gisev N, Bell JS, Korhonen MJ, Ilomäki J. Predictors of persistent prescription opioid analgesic use among people without cancer in Australia. Br J Clin Pharmacol. 2018;84:1267-78.

49. Inacio MC, Hansen C, Pratt NL, Graves SE, Roughead EE. Risk factors for persistent and new chronic opioid use in patients undergoing total hip arthroplasty: a retrospective cohort study. BMJ Open. 2016;6:e010664.

50. Seçil Y, Arıcı Ş, İncesu TK, Gürgör N, Beckmann Y, Ertekin C. Dysphagia in Alzheimer's disease. Neurophysiol Clin. 2016;46:171-8.

51. Pergolizzi J, Boger RH, Budd K, Dahan A, Erdine S, Hans G, Kress HG, Langford R, Likar R, Raffa RB, Sacerdote P. Opioids and the management of chronic severe pain in the elderly: consensus statement of an International Expert Panel with focus on the six clinically most often used World Health Organization Step III opioids (buprenorphine, fentanyl, hydromorphone, methadone, morphine, oxycodone). Pain Pr. 2008;8:287-313.

52. Grond S, Sablotzki A. Clinical pharmacology of tramadol. Clin Pharmacokinet. 2004;43:879-923.

53. Puranen A, Taipale H, Koponen M, Tanskanen A, Tolppanen AM, Tiihonen J, Hartikainen S. Incidence of antidepressant use in community-dwelling persons with and without Alzheimer's disease: 13-year follow-up. Int J Geriatr Psych. 2017;32:94-101.

54. Erdal A, Flo E, Aarsland D, Selbaek G, Ballard C, Slettebo DD, Husebo BS. Tolerability of buprenorphine transdermal system in nursing home patients with advanced dementia: a randomized, placebo-controlled trial (DEP. PAIN. DEM). Clin Interv Aging. 2018;13:935-46.

55. Erdal A, Flo E, Aarsland D, Ballard C, Slettebo DD, Husebo BS Efficacy and safety of analgesic treatment for depression in people with advanced dementia: randomised, multicentre, doubleblind, placebo-controlled trial (DEP. PAIN. DEM). Drugs Aging. 2018;35:545-58.

56. Wettermark B, Zoëga H, Furu K, Korhonen M, Hallas J, Nørgaard M, Almarsdottir A, Andersen M, Andersson Sundell K, Martikainen J, Mortensen M, Petzold M, Wallach-Kildemoes H, Wallin C, Sørensen H. The Nordic prescription databases as a resource for pharmacoepidemiological research—a literature review. Pharmacoepidemiol Drug Saf. 2013;22:691-9. 